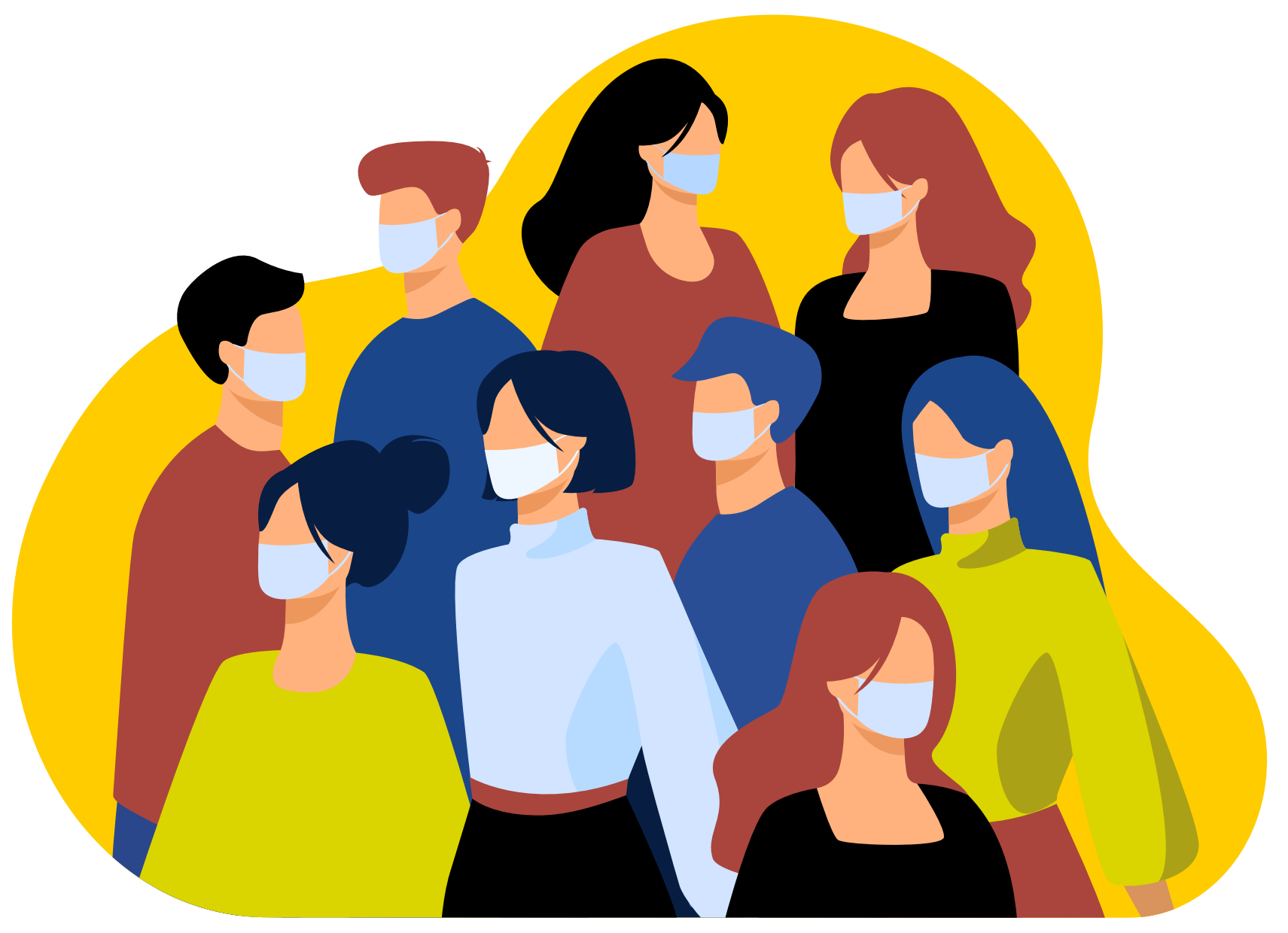

\title{
Cuando llegue la hora de volver
}

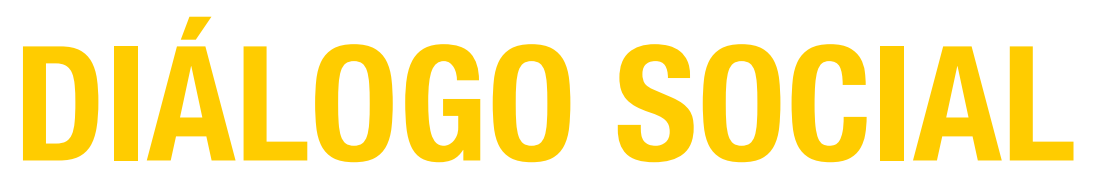

\section{y la organización del trabajo para salir de la crisis}

La prudencia extrema en los ritmos del proceso - en la medida que el objetivo no es el crecimiento, sino evitar el shock-, el diálogo entre los actores, el carácter compartido de los sacrificios y un estado velando por los derechos fundamentales de trabajadores y trabajadoras son algunos elementos indispensables de considerar.

Eduardo Abarzúa C. Ph.D en Ciencias del Trabajo, Universidad Católica de Lovaina. Decano FEN- UAH.

Yerko Ljubetic G. Máster en Políticas del Trabajo y Relaciones Laborales en Alma Mater Studiorum, Universitá de Bologna. Profesor Magíster en Gestión de Personas en Organizaciones UAH. Consejero Instituto Nacional de Derechos Humanos.

in perjuicio de que formamos parte de aquellos que consideran que una convocatoria a cualquier modalidad de activación de una cierta normalidad debe estar condicionada al logro de niveles sanitarios que garanticen la seguridad y salud de las personas, situación que parece aún estar lejos de nuestra realidad, es evidente que las características de nuestra economía y de la sociedad han impues- to una presión creciente por retomar la actividad económica que probablemente vaya siendo progresivamente acatada por la mayoría de las personas que dependen de sus ingresos contingentes y diarios para sobrevivir, sin embargo, los hechos nos demuestran que siempre la mejor economía será la salud pública.

En tales circunstancias, se impone la necesidad de debatir respecto de las medidas que deben disponerse para establecer una forma de organización del trabajo que priorice la salud de trabajadores y trabajadoras por sobre cualquier otra consideración. Si bien la dimensión abordada es sustantiva, preocupa que la discusión sea sólo técnica (flujos de trabajadores, diseño de turnos, organización del trabajo, etc.) y unilateral o dirigida desde el gobierno y actores empresariales sin consideración ni escucha de los 
trabajadores y sus organizaciones, cuestión que ya ocurrió cuando se convocó a los trabajadores del sector público. Al respecto, existen aspectos ineludibles sin los cuales cualquier perspectiva de organización del trabajo adecuada a las circunstancias se vuelve inviable.

\section{EN PRIMER LUGAR, EL DIÁLOGO SOCIAL}

El que la determinación de modalidades de trabajo presencial sea producto del diálogo entre empleadores y trabajadores y sus organizaciones es una condición ética vinculada no sólo al riesgo que estos últimos deben asumir, el que, por supuesto, incluye el traslado hacia y desde sus lugares de trabajo, sino también a su eficacia práctica, en la medida que el concurso del mayor conocimiento concreto sobre las particularidades de cada actividad es imprescindible para cada decisión que se deba adoptar. Los tiempos y modalidades en las que cada persona desempeñará sus labores en un contexto de riesgo para su salud no pueden ser decididas de un modo unilateral y desde las seguridades de las oficinas gerenciales o del gobierno central, sino en diálogo abierto y dinámico con quienes deberán asumir ese riesgo.

Este diálogo debe darse no solo a nivel de empresa o unidad productiva, sino que debe implicar el involucramiento de las organizaciones sindicales de grado superior y sus equivalentes empresariales, de manera de ir apoyando los esfuerzos específicos y dando alcance más amplio a las conclusiones y hallazgos a los que en conjunto se arribe, así como llamando a las autoridades a adoptar las medidas que sean necesarias en el ámbito público. Salir "mejor que antes" de la pandemia requiere reforzar los niveles de articulación y negociación entre los actores productivos, así (re)construimos tejido social.

Lamentablemente, la recientemente aprobada ley de teletrabajo no consideró los pactos de adaptabilidad laboral para el ajuste de los contratos de trabajo y, dejó a la asimetría de las partes, el arreglo de las condiciones de trabajo a la nueva realidad.

\section{EN SEGUNDO LUGAR, LA RECIPROCIDAD}

No es novedoso afirmar que las relaciones laborales en nuestro país están marcadas por la desconfianza entre sus actores. Más allá de profundizar en las razones de ello, es claro que esta característica no puede ser eludida a la hora de encarar el desafío de diseñar formas de organización del trabajo que puedan coexistir con el riesgo sanitario.
En efecto, es altamente probable que la convocatoria a "una nueva normalidad" laboral sea vista por la mayor parte de trabajadores y trabajadoras como inspirada en finalidades sólo orientadas a mantener los beneficios de las empresas y sus propietarios. En el contexto de desigualdad que caracteriza a nuestra sociedad, esa aprensión aparece como plausible y se transfiere a la institucionalidad laboral, comprometiendo las posibilidades de abordar de manera adecuada el problema.

Así, se hace necesario que el proceso de diálogo sugerido contenga las modalidades a través de las que se pueda constatar que el esfuerzo que se acomete es compartido por los actores, tanto en términos de las tareas concretas a asumir,

\section{"La recientemente aprobada ley de teletrabajo no consideró los pactos de adaptabilidad laboral para el ajuste de los contratos y dejó a la asimetría de las partes el arreglo de las condiciones de trabajo a la nueva realidad"}

como de los beneficios que irrogue el eventual éxito de éstas.

La apreciación de que este desafío se hace descansar, una vez más, sólo sobre las espaldas de los más débiles, además de asegurar el fracaso, ahondaría la crisis de confianza y credibilidad que, como hemos dicho, caracteriza las relaciones en nuestro mundo del trabajo. Cuando las relaciones en el trabajo (y en la sociedad) son asimétricas, quien posee más recursos y poder debe preguntarse por sus deberes de cara a los más débiles.

\section{ENTERCER LUGAR, EL ROL DEL ESTADO}

Tratándose de resolver los modos en que se retoma la actividad productiva en el contexto de crisis sanitaria, el carácter naturalmente desigual de las relaciones entre trabajadores y empleadores cobra, como es obvio, una especial trascendencia. De ello se debe concluir necesariamente que el rol del estado y los organismos públicos debe reforzarse en la perspectiva de proteger los derechos fundamentales de trabajadores y trabajadoras, estimulando la cohesión social y no una idea individualista de solución.

En tal sentido, las medidas que se tomen no pueden construirse desde el supuesto que concurren actores iguales en sus capacidades de negociación y de resolver los problemas de la crisis. Por lo mismo, debe acordarse un marco normativo que desarrolle y profundice las obligaciones del empleador respecto a la salud e integridad física y sicológica de sus dependientes, así como la drasticidad de las sanciones por su incumplimiento y las capacidades fiscalizadoras de los organismos competentes.

Por otro lado, deben precisarse las funciones de entidades como las mutuales de seguridad en las labores de prevención, capacitación y atención médica de quienes resulten afectados, la incorporación obligatoria de disposiciones específicas en los reglamentos internos de higiene y seguridad, así como establecer disposiciones que no dejen lugar a dudas, en el ámbito tanto administrativo como jurisdiccional, sobre la pertinencia y financiamiento de las licencias médicas a que haya lugar.

El estado, en todas sus expresiones, no puede ser ajeno a los avances y definiciones que se vayan adoptando. Por el contrario, debe estar atento al riesgo de quienes se ven obligados a volver al trabajo por razones de subsistencia. Se trata de derechos humanos finalmente $y$, por ello, debe generar un entorno de protección efectiva que evite que trabajadores y trabajadoras expongan la salud y la vida por un imperativo económico.

La manera en que esta urgente materia sea abordada y resuelta será especialmente determinante en una sociedad a la que la pandemia sorprendió en medio de una grave crisis social y política. Por ello, la prudencia extrema en los ritmos del proceso -en la medida que el objetivo no es el crecimiento, sino evitar el shock-, el diálogo entre los actores, el carácter compartido de los sacrificios y un estado velando por los derechos fundamentales de trabajadores y trabajadoras son algunos elementos indispensables de considerar.

En lo que hagamos hoy, se juega parte importante de la sociedad que seremos una vez superada la crisis y las instituciones de educación superior tenemos que involucrarnos y estar en condiciones y disponibilidad para colaborar, generando y entregando conocimientos e iniciativas que vayan en línea de la construcción de más y mejor cohesión social. $\mathbf{E}$ 